\title{
Ciclo de conversión de efectivo: una herramienta esencial para la evaluación financiera de la empresa
}

\author{
Cash conversion cycle: an essential tool for the financial evaluation of the \\ company
}

\author{
Juan Bernardo Morillo Rodriguez ${ }^{1 *}$, Denis Ivan Llamo Santa Cruz ${ }^{1}$ \\ ${ }^{1}$ Escuela Profesional Contabilidad, Facultad de Ciencias Empresariales, Universidad Peruana Unión
}

INFORMACIÓN DEL ARTÍCULO

Historia del artículo

Recibido: 18 de junio 2019

Aceptado: 20 de agosto 2019

\section{Palabras clave:}

Ciclo de conversión efectivo, cuentas por pagar, inventarios, cuentas por pagar, rentabilidad, liquidez
Keywords:

Cash conversion cycle, accounts payable, inventories, accounts payable, profitability, liquidity

\section{Resumen}

El presente artículo estudia el ciclo de conversión de efectivo como herramienta indispensable para la evaluación financiera de la empresa. El ciclo de conversión de efectivo indica el tiempo que toma desde que se compra la mercadería, se vende y se vuelve a cobrar. Existe la posibilidad para aumentar la rentabilidad mediante la reducción del período de recolección de cuentas por cobrar, disminuyendo el período de conversión de inventarios, y alargando el período de pago. Por ello, cuanto más corto sea su ciclo de conversión de efectivo mayor liquidez tendría la entidad; por otro lado, una baja rotación en las cuentas por cobrar indica que la empresa está tardando mucho en recuperar sus ventas a crédito, de allí que, el éxito de la empresa también depende de la eficacia de la gestión de su inventario. Por lo tanto, toda empresa debe tener en cuenta que el nivel de rotación de los inventarios, refleja el avance de las ventas proyectadas, variando, según el tipo de empresa, siendo diferentes tanto en la producción como en las ventas; un claro ejemplo se tiene en la comparación entre un supermercado y una empresa de camiones. Asimismo, la rotación de cuantas por pagar muestra el tiempo necesario para liquidar los pagos de cuentas. Por ello, un pago rápido permite a la empresa disfrutar del descuento y mantener una buena reputación con los proveedores.

\section{Abstract}

This article studies the cash conversion cycle as an indispensable tool for the financial evaluation of the company. The cash conversion cycle indicates the time it takes since the merchandise is purchased, sold and re-collected. There is the possibility to increase profitability by reducing the collection period of accounts receivable, reducing the inventory conversion period, and extending the payment period. Therefore, the shorter its cash conversion cycle, the more liquidity the entity would have; On the other hand, a low turnover in accounts receivable indicates that the company is taking a long time to recover its sales on credit, hence, the success of the company also depends on the effectiveness of managing its inventory. Therefore, every company should keep in mind that the level of inventory turnover reflects the progress of projected sales, varying, depending on the type of company, being different in both production and sales; a clear example is the comparison between a supermarket and a trucking company. Also, the turnover of how many payable shows the time needed to settle account payments. Therefore, a quick payment allows the company to enjoy the discount and maintain a good reputation with suppliers. 


\section{Introducción}

Las empresas grandes y pequeñas deben conocer la importancia de la realización de un análisis financiero en sus actividades empresariales (Porlles, Quilolspe, y Salas, 2013). Por lo tanto, existen varias formas de realizar un análisis financiero, todo dependerá del área que se desee evaluar, en este caso se habla del análisis al ciclo de conversión de efectivo.

El propósito de la base de todas las empresas es ser lucrativo y tener suficiente dinero para pagar sus obligaciones a corto plazo y mejor rendimiento de la compañía (Nazir, Khan, y Khan, 2016). Es por ello, que toda entidad busca eficacia en la gestión de inventarios, cuentas, por pagar, cuantas por cobrar y el efectivo, así como también una buena administración del efectivo para no tener la necesidad de financiación. Por lo tanto, el rendimiento de toda entidad dependerá principalmente de la buena gestión de sus recursos en todo momento.

Una empresa debe conocer el tiempo que los lleva en promedio para convertir sus bienes y servicios en dinero en efectivo. Este periodo de tiempo se conoce como ciclo de conversión de efectivo (Zakari y Saidu, 2016); indicando la longitud del periodo entre el pago de proveedores y ser pagados por los clientes; teniendo como determinantes, los días de proveedores pendientes, días de inventario y días de crédito restante.

En otras palabras, el ciclo de conversión de efectivo es la cantidad de tiempo efectivo de una empresa que está atado en el capital de trabajo antes de que el dinero sea finalmente devuelto o cuando los clientes paguen por los productos o servicios prestados (Calle, 2016).
Según Bermeo (2017) y Jiménez con Rojas (2013), el ciclo de conversión del efectivo nos indica el tiempo que toma desde que se compra la mercadería, se vende y se vuelve a cobrar; el fallo en una de las tres áreas puede causar reducción del efectivo debido a que el plazo de las cuentas por cobrar fueron extendidos; así como también una menor rotación afecta el inventario de mercadería y las cuentas por cobrar tendrán la necesidad de ser financiadas con endeudamiento a corto plazo.

El ciclo de conversión de efectivo puede ser positivo o negativo. Por ello, se considera que cuando el ciclo de conversión del efectivo es positivo, indica el número de días que la gestión de la empresa debe organizar prestar fondos y recurrir a sus activos líquidos disponibles antes de que se les coleccione de sus cuentas por cobrar; pero si el ciclo de conversión del efectivo fuese negativo se considera muy beneficioso para la empresa debido a que ha recibido de sus deudores antes de que tenga que cumplir con sus obligaciones con sus acreedores (Muneeb y Kashif, 2012).

De allí que muchas veces el ciclo de conversión de efectivo suele variar según el rubro de la empresa ya que ello dependerá la rotación de sus cuentas. Mientras que las cuentas por cobrar y los inventarios sean liberados en lo más posible, la entidad tendrá dinero en efectivo para invertir en otras vías más rentables y mantener una ventaja competitiva en la industria.

Según Abadillo (2011), los resultados del ciclo de conversión de efectivo, determinan las necesidades de captación de recursos de una empresa, debido a que los días que se retienen los recursos den- 
tro de los inventarios y cuentas por cobrar, deben obtenerse de los proveedores, de lo contrario tendrá que recurrirse a llamamientos de capital o endeudamiento externo.

Por ello es muy importante realizar un diagnóstico financiero, ya que evalúa la salud financiera de la empresa, así como los resultados de sus operaciones, con el fin de determinar una estimación sobre su situación y sus resultados futuros, posibilitando tomar decisiones eficientes que garantizan el empleo nacional de los recursos materiales y financieros de la empresa.

Por tal motivo la presente investigación tiene como objetivo dar a conocer el ciclo de conversión de efectivo como una herramienta financiera para la empresa; estudio que nos permitirá conocer la eficacia dentro de la empresa. El documento está estructurado por los fundamentos teóricos sobre el ciclo de conversión de efectivo y sus respectivos componentes.

\section{Metodología}

La metodología tuvo como base de estudios, diferentes fuentes documentales que contienen artículos publicados en revistas de reconocida trayectoria. Esta revisión documental solo se centró en los artículos publicados en revistas del área de finanzas divulgado por las universidades, página web y bibliotecas locales; así como también se recurrió a la base de datos Scielo, Google académico Redalyc, Dialnet, etc.

Se encontraron 25 artículos y se analizaron cada uno de ellos, especialmente los 20 artículos que estaban relacionados con el objeto de estudio (García, Galarza, y Altamirano, 2017). Una vez definido el objeto de estudio y de hacer una lectura analítica de los textos para conocer ideas que orienten el trabajo de investigación; fue necesario establecer algunos criterios de ordenación y clasificación de la información. Para ello, se creó una base de datos en Excel definiendo los siguientes campos: título del artículo, autor, año de publicación, nombre de la revista.

Razón por la cual, sirvió para una mejor preparación en el desarrollo del articulo; teniendo una iniciativa con la caracterización del ciclo de conversión de efectivo, sus componentes, la revisión de las investigaciones y finalmente sus conclusiones.

\section{Revisión de la literatura}

\section{Ciclo de conversión de efectivo}

Según Manuebb y Kashif, (2012), ciclo conversión de efectivo se ha considerado una medida útil para la administración del capital de trabajo de la empresa y sobre todo la gestión de efectivo.

Este se determina a partir del tiempo necesario para la compra de materias primas, pasando por la fabricación, hasta recoger el dinero de la venta de las mercadería vendida (Nguyen y Sundaresan, 2018).

Asimismo, el ciclo de conversión en efectivo se mide mediante la deducción del período de pago a proveedores del total de período de conversión de inventarios y cuentas por cobrar periodo de recogida.

Por ello, tiene el mérito de integrar el análisis de tres importantes cuentas del balance en las empresas comerciales (inventarios, cuentas por cobrar a clientes y cuentas por pagar a proveedores. (Moreno, 2004)

Según estudios realizados se demuestra que existe la posibilidad de reducir el 
ciclo de conversión de efectivo a través de la reducción del inventario y la reducción en el número de días pendientes de cobro (Ali y Atif, 2012).

Asimismo, ofrece de forma fácil y útil para comprobar la gestión del capital de trabajo. También indica que las empresas puedan reducir su ciclo de conversión de efectivo para aumentar la rentabilidad.

Esto se puede hacer mediante la reducción del período de recolección de cuentas por cobrar, disminuyendo el período de conversión de inventarios, y alargando el período de pago. (Nguyen y Sundaresan, 2018)

Por otro lado Ali y Atif, (2012) indican que la colección lenta de las cuentas por cobrar se correlaciona con una baja rentabilidad; así como también la gestión eficiente de los inventarios mejora la rentabilidad de las empresas.

De acuerdo con este cuerpo de literatura un ciclo de conversión en efectivo más corto indica que una empresa gestiona el flujo de efectivo de manera eficiente, ya que genera más ventas por unidad de capital invertido; razón por lo cual, conducirá a una mayor rentabilidad (Emmanuel y Agyapong, 2016).

Es por ello, cuanto más corto sea su ciclo de conversión en efectivo , mayor liquidez tendría la firma que adquiere regularmente mercancías para su venta al público (Moreno, 2004). Asimismo tendrá una menor necesidad de financiamiento externo durante el mismo ciclo (Moreno, 2004).

Según, Nguyen y Sundaresan, (2018) el estudio de ciclo de conversión en efectivo es variable de acuerdo a la actividad empresarial a realizar, es por ello que una empresa comercial va tener el ciclo de conversión en efectivo en negativo, por el mismo hecho que sus actividades fluyen diariamente y los productos que se tiene son ya terminados, a comparación con una empresa industrial su ciclo de conversión en efectivo va hacer positivo, porque el proceso para obtener el producto terminado va a demandar de mayor tiempo en elaboración.

De tal manera para acortar el ciclo de conversión en efectivo en una firma como se aprecia en la figura 1 , se debe primero disminuir el periodo de rotación de inventarios, que se obtiene analizando los procesos de producción para así identificar los componentes innecesarios para dicho proceso de producción; esto con el propósito de fabricar los productos de manera eficiente.

Asimismo, en una empresa comercial, lo que se busca es reducir los tiempos de viaje y de llegada de la nueva mercancía también reduciendo el tiempo en almacenamiento.

Segundo se debe disminuir el periodo de cobranza es por ello que se debe tener en cuenta dos cosas: la relación de clientes al contado, creando un segmento estratégico de cobro, hay que tener en cuenta los clientes al crédito, asignando descuentos en sus deudas, políticas de pago, con el fin de motivar a los clientes y cancelen incluso antes del plazo.

Por otro lado, se debe aumentar el periodo de las cuentas por pagar, negociando el crédito con los proveedores, sin llegar a perjudicar la misma imagen y reputación crediticia de la empresa. 


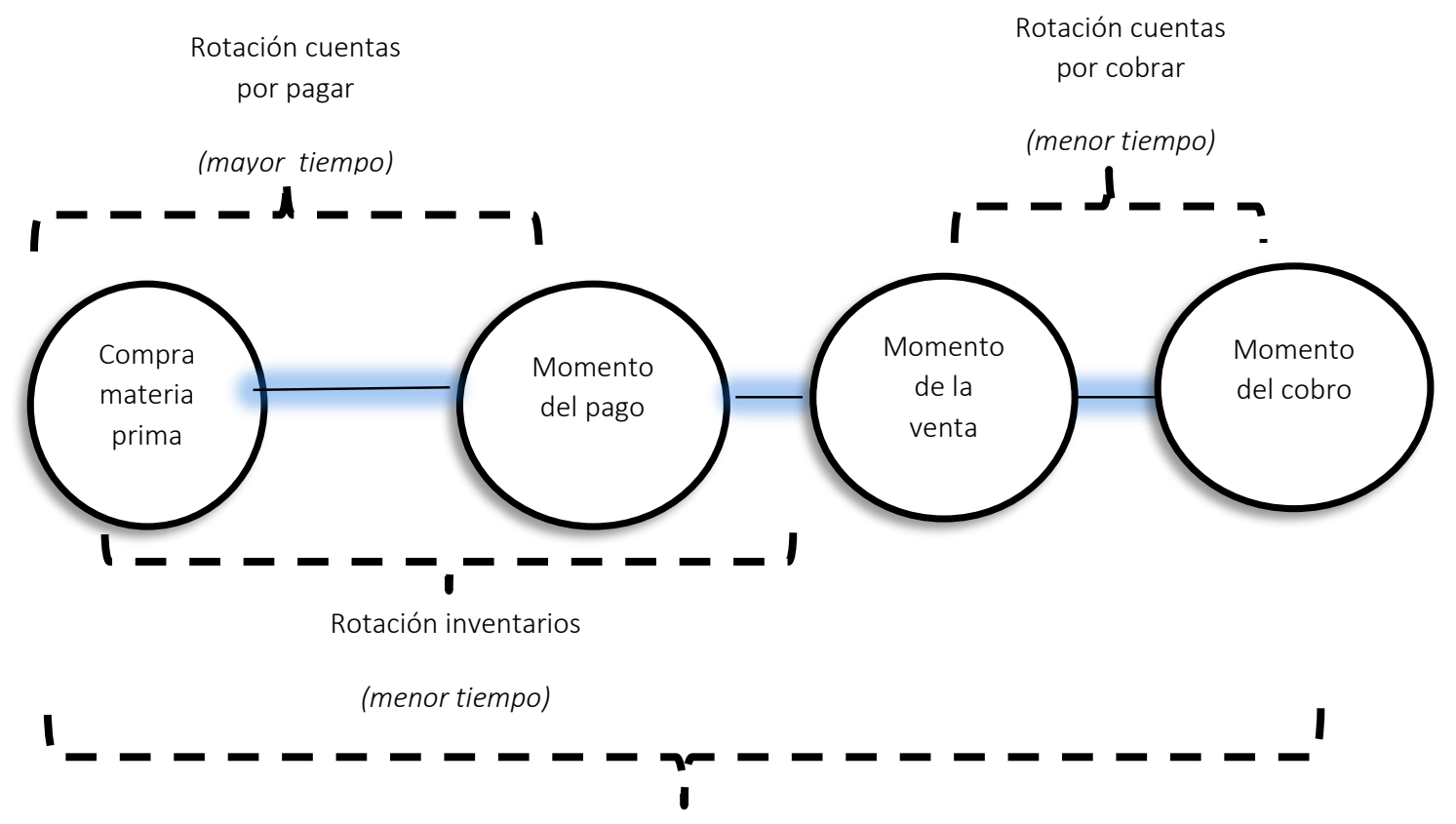

Figura 1: ciclo de conversión en efectivo Fuente: elaboración propia

Rotación de cuentas por cobrar

Según Ghapar, (2018) este ratio financiero hacer referencia al tiempo necesario para recoger dinero en efectivo de los clientes. Por ello, el éxito de una empresa depende de la gestión de los derechos de cobro en el efectivo.

Es decir, si la empresa realiza una recolección rápida de las cuentas por cobrar, no puede tener problemas de caja para los costos de operación. Gutiérrez (2012) menciona que el objetivo principal de administrar las cuentas por cobrar es cobrarlas tan rápido como sea posible sin perder ventas.

Asimismo Barboza (2016) indica que una alta rotación revela que las ventas a crédito se recuperan de forma rápida, lo cual da liquidez a la empresa.
Por otro lado, una baja rotación indica que la empresa está tardando mucho en recuperar sus ventas a crédito.

Del mismo modo lo reconoce (Muñoz, 2013), al señalar que es deseable que el saldo de cuentas por cobrar rote razonablemente, de tal manera que no implique costos financieros muy altos y permita utilizar el crédito como estrategia de ventas (Graziano, 2011).

Para su mejor comprensión y gestión, la rotación de cuentas por cobrar está en función a los términos y condiciones establecidas para la cobranza, así como los límites de crédito el factoring y la actualización de los datos de los clientes, como se aprecia en la figura 2. 


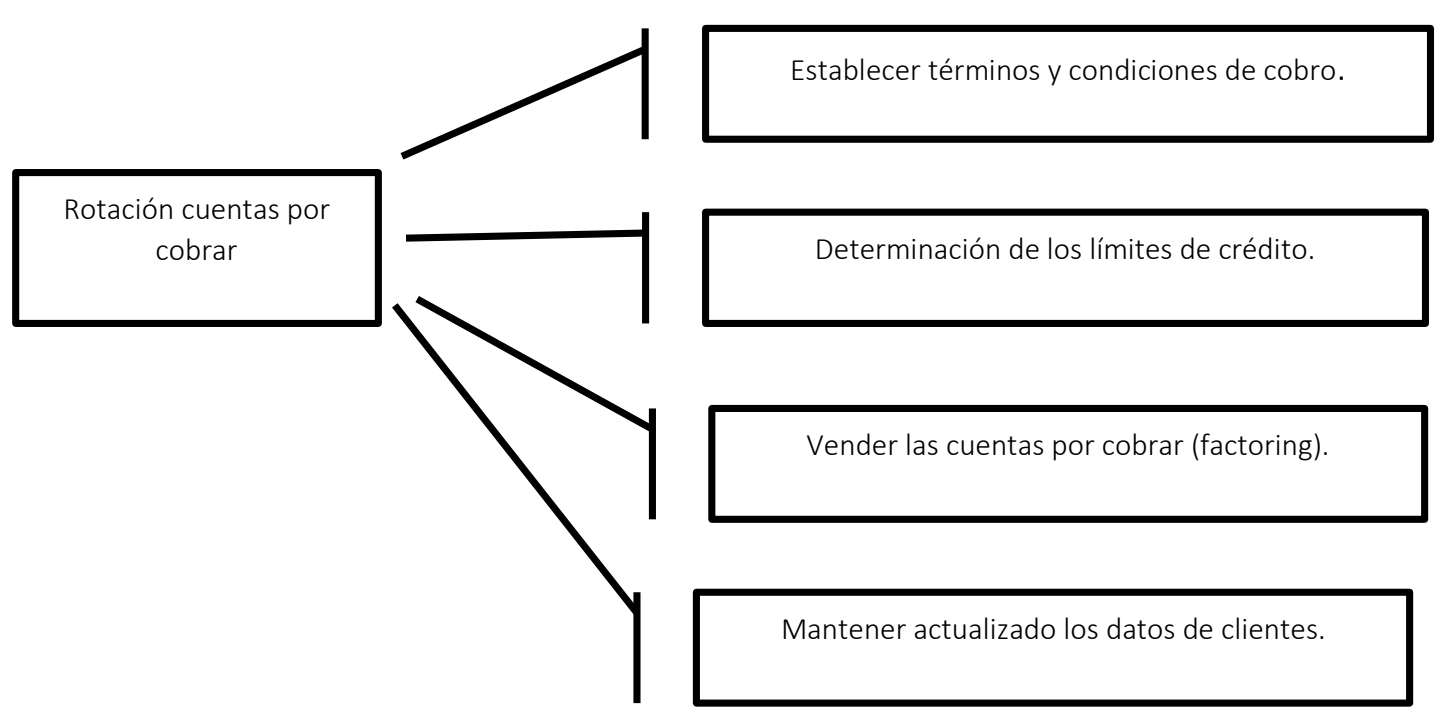

Figura 2: rotación cuentas por cobrar

Fuente: elaboración propia

\section{Inventarios}

Tal como se menciona en la rotación de cuentas por cobrar, el éxito de la empresa también depende de la eficacia de la gestión de su inventario (Ghapar, 2018). Del mismo modo, una proporción baja rotación, implica ventas pobres y el exceso de inventario. (Bus, 2016)

Actualmente existe una preocupación constante por las políticas de inventario por parte de las empresas; debido a que el costo del dinero que se utiliza para comprar y mantener inventarios es de un $15 \%$ aproximadamente correspondiente a un gran número de empresas en economía de mercado de determinado nivel de desarrollo. (Ceballos, 2015)

Según Ceballos (2015), los inventarios son esenciales para la venta, y las ventas necesarias para las utilidades. Es por ello que, en la actualidad, un gran número de empresas están utilizando modelos computarizados para llevar a cabo el con-

trol de los inventarios con la finalidad de coordinar los inventarios disponibles con los niveles pronosticados de ventas; así como también mantienen una coordinación más estrecha con sus proveedores a efecto de reducir los niveles promedio de inventarios.

Según Luis, (2016) las existencias están compuestas por las partidas de los inventarios que posee la empresa en materias primas, productos terminados en proceso y productos terminados.

Asimismo, el inventario es considerado como una inversión debido a que se espera un rendimiento en el corto plazo por el desembolso de efectivo realizado al comprar la materia prima.

Por su parte, Arango (2014) y Yera (1999) sostienen que los inventarios son bienes destinados principalmente para la venta en forma directa o indirecta, donde se evalúan al costo o al valor neto realizable (Muñoz, 2013). 
Para Gutiérrez (2012) esto significa la suma de aquellos artículos tangibles de propiedad personal los cuales están disponibles para la venta en una operación ordinaria comercial y están en un proceso de producción para tales ventas, que es el tiempo que permanece en la bodega hasta venderse (Barboza, 2016).

Sánchez (2011), menciona que la rotación del inventario mide el promedio durante todo el año, además de mostrar la rapidez en que el inventario se convierte en cuentas por cobrar o en efectivo.

A su vez Manchego (2016) establece que indica con qué frecuencia sale una existencia del almacén para ser vendida, con lo cual se debe propiciar que tenga un elevado nivel de rotación.
Para Ponce (2014), es controlar las salidas de referencia y cantidades del centro de distribución y se calcula indica el número de veces que el capital invertido se recupera a través de las ventas.

Por tanto, la rotación de los inventarios refleja el avance de las ventas proyectadas, por ello, suele variar según el tipo de empresa; por ejemplo un supermercado con una empresa de camiones tendrán mucha diferencia en la rotación de inventarios.

Sin embargo, ambas pueden calcular y controlar sus inventarios mediante la utilización de un sistema óptimo de inventarios, el sistema $A B C$, JIT, o stock fijo, como se detalla en la figura 3 .

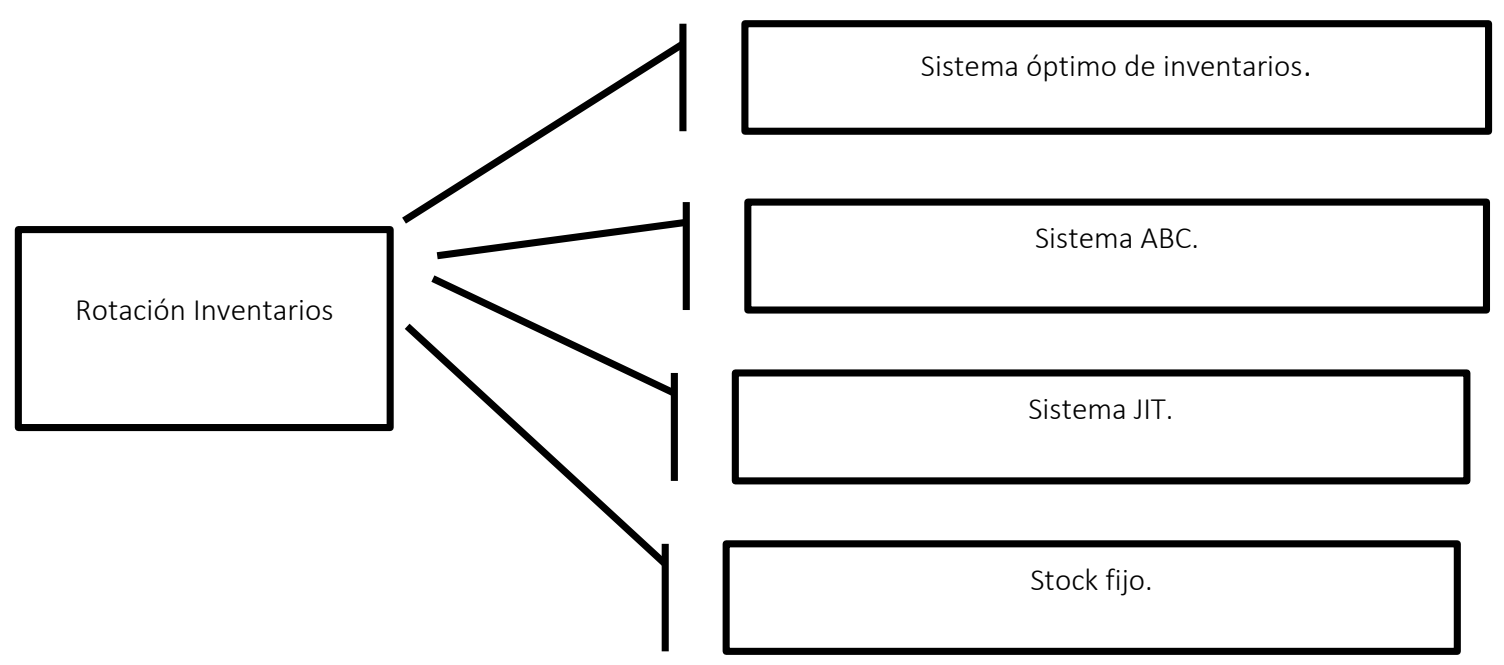

Figura 3: Rotación inventarios

Fuente: elaboración propia 


\section{Rotación de cuentas por pagar}

La rotación de cuantas por pagar es el tiempo necesario para liquidar los pagos de cuentas. Por ello, un pago rápido permite a la empresa disfrutar del descuento y mantener una buena reputación con los proveedores.

Por otro lado, retrasar el pago de las cuentas por pagar, permite utilizar el dinero para generar beneficios, pero puede poner en peligro la relación con el proveedor y perdió el descuento por pronto pago.

De tal forma que ambos enfoques pueden tener impacto en el rendimiento financiero de la compañía (Nobanee, 2018).

Según Akoto, Awunyo, Lawer, (2013) este ratio se calcula de la siguiente forma: promedio de las cuentas, dividido por el costo de ventas multiplicado por 365 días.

Se espera que las obligaciones se relaciones positivamente con la rentabilidad de la empresa. Es decir, mientras aumenten los días de las cuentas por pagar la empresa tiende más tiempo a reinvertir y ganar por los intereses del dinero de los acreedores o se utilizaría para la adquisición de otros activos a corto plazo.

Barboza (2016) y Guzmán (2006) consideran que este indicador se debe de contrastar con el plazo que brindan los proveedores a la empresa, si el plazo es mayor a lo pactado.

Para Yera (1999) significa que la empresa no cumple con los términos estipulados y puede deteriorar la capacidad de su financiamiento con los proveedores.

Asimismo Valero (2013) sugiere que hay diversas fomas de pulir el efectivo a través de las cuentas por pagar disponiendo los pagos lo más cerca posible a la fecha de cumplimiento, adaptando todas las reducciones viables y custodiar que los pagos sean perpetrados a través de cheques (Graziano, 2011).

Por tanto, para mejorar la rotación de cuentas por pagar, debe tener en cuenta el aprovechar los plazos de pago que ofrecen los proveedores, así como elegir a los proveedores más allá de los precios debiendo asegurar que los pagos se realicen a tiempo, como se verifica en la figura 4 .

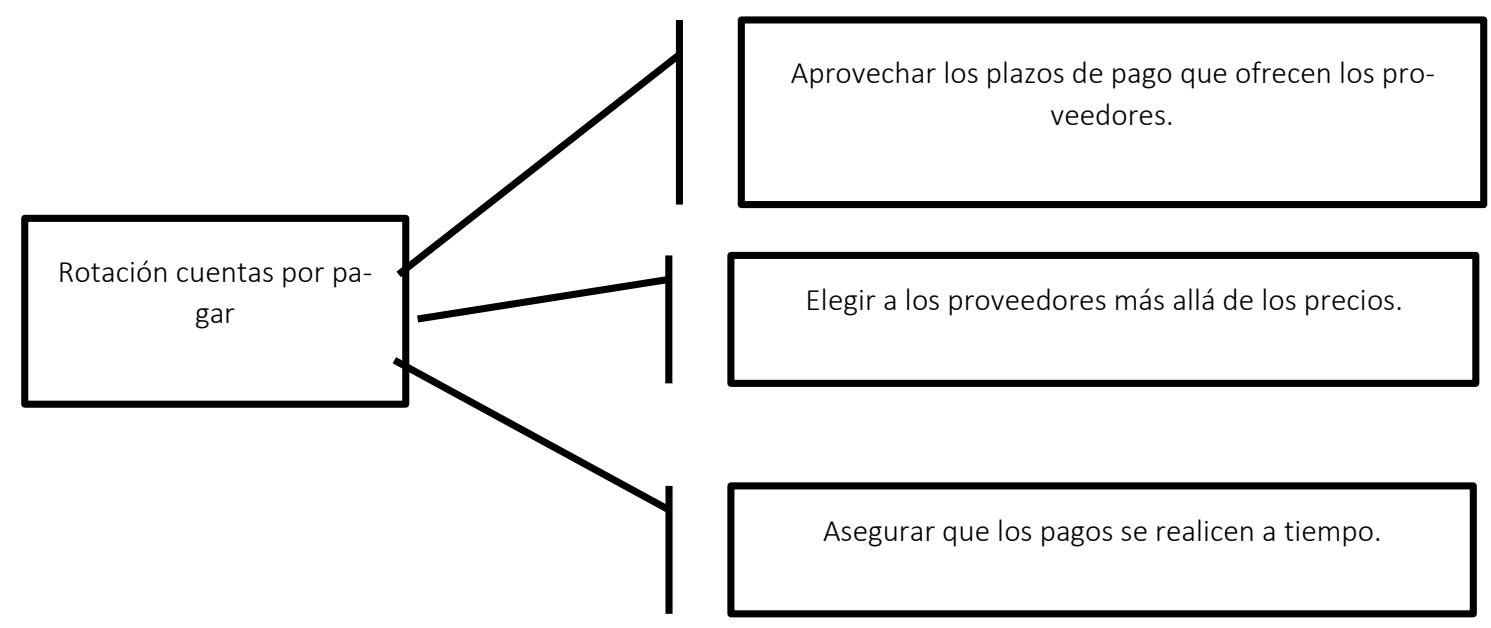

Figura 4: rotación de cuentas por pagar Fuente: elaboración propia 


\section{Conclusión}

Para obtener una liquidez eficiente en una firma, es de vital importancia el manejo del ciclo de conversión en efectivo, para ello la gerencia debe gestionar eficientemente los tres componentes como lo son, cuentas por cobrar que deben ser a corto plazo, cuentas por pagar que deben ser a largo plazo e inventarios a corto plazo.

Por lo tanto, mientras el ciclo de conversión de efectivo sea negativo o menor, aumenta la rentabilidad; una entidad con una liquidez solida no va necesitar prestamos de terceros ya que puede financiarse con sus propios recursos.

Es por ello, que el ciclo de efectivo suele variar según a la operatividad de la empresa; tal es el caso en las entidades comerciales que tienen la rotación de inventarios más acelerada; por el mismo hecho que los productos a vender son ya terminados y es por ello que el resultado del ciclo de efectivo refleja un indicador a corto plazo; a comparación de empresas industriales siendo su rotación del ciclo de efectivo extenso ya que el proceso de elaboración del producto terminado demanda de mayor tiempo, es por ello que el ciclo de efectivo genera resultados con indicadores a largo plazo.

Asimismo, las investigaciones demuestran que los gerentes pueden crear valor reduciendo los días de cartera y los días de inventarios. Por otro lado, el ciclo de conversión de efectivo es de vital importancia en la rentabilidad económica ya que determina liquidez a corto plazo debido al manejo adecuado de la rotación de inventarios, lo cual se obtendrá una eficiente venta, generando un porcentaje favorable de rentabilidad económica para la entidad. 


\section{Referencias}

Abadillo, A. (2011). Administración del ciclo de conversión de efectivo en las empresas comercializadoras de neumáticos en Guatemala.

Akoto, R. K., Awunyo, vitor D., y Lawer, P. (2013). La gestión del capital y la rentabilidad: Evidencia de las empresas manufactureras de Ghana cotizadas, 5(9), 373-379.

Ali, A., y Atif, S. (2012). Trabajando en la gestión de capitales.

Arango, M. (2014). Estrategias de Control de Inventarios para optimizar la produccion y Rentabilidad de la empresa Agro Macathon S.A.C. Universidad Autonoma del Peru.

Barboza, M. (2016). Propuesta de un Modelo para la valoracion financiera del patrimonio de la empresa INTESOFT S.A. universidad de Costa Rica.

Bermeo, J. (2017). Analisis del ciclo operativo y su importancia en el estudio del rendimiento economico y financiero de una empresa de servicios. Universidad Tecnica de Machala. https://doi.org/1390-9304

Bus, A. M. (2016). Articulo de revisión de la gestión de negocios y bebidas en Nigeria.

Calle, J. (2016). Diseño de estrategias para el ciclo de conversiòn de efectivo de la empresa superglam. universidad estatal de milagro.

Ceballos, S. (2015). La importancia de las estrategias para la administración eficiente del efectivo. Observatorio De La Economia Latinoamericana, (210), 1-19.
Emmanuel, K., y Agyapong, G. (2016). Teoría ciclo de conversión de efectivo y la rentabilidad de las empresas: Evidencia de las empresas no financieras cotizadas en el Johannesburgo, 6(3), 37-51.

García, J., Galarza, S., y Altamirano, A. (2017). Importancia de la administración eficiente del capital de trabajo en las Pymes. Ciencia UNEMI, 10(c), 30-39. Retrieved from http://ojs.unemi.edu.ec/ojs/index.php/cie nciaunemi/article/view/495

Ghapar, F. A. (2018). Gestión de Operaciones y de su efecto sobre Rentabilidad: evidencia empírica de mercado de capitales de Malasia.

Graziano, M. (2011). Ciclo de Conversion del Efectivo en la Inversion yLiquidez en las empresas Picadoras de Piedra Calizada del Municipio Rosario de Perija, (V), 141.

Gutierrez, I. (2012). Mejoramiento de la Gestion del Capital de Trabajo y del Ciclo de Efectivo, en la Empresa NR Productos Industriales y Agricolas S.A., Ubicada en la Provincia. Pontificia Universidad Catolica del Ecuador.

Guzmán, A. (2006). Ratios Financieros y Matematicas de la Mercadotecnia (series myp). lima.

Jiménez, J., y Rojas, F. (2013). The importance of cash cycle and calculation of working capital management in smes. Clío América, 7(13), 48-63. Retrieved from

http://revistas.unimagdalena.edu.co/inde x.php/clioamerica/article/view/436

Manchego, T. (2016). Análisis Financiero Y La Toma De Decisiones En La Empresa Clinica Promedic S.Civil.R.L. Universidad Privada de Tacna. 
Manuebb, A., y Kashif, R. (2012). La relación óptima del ciclo de conversión de efectivo con Tamaño de la empresa y la rentabilidad, 2(4), 189-203.

Moreno, N. (2004). Ciclo De Conversión De Efectivo En Las Grandes Empresas De Comercio Al Detal En Colombia. Estudios Gerenciales, (1980), 129-140.

Muneeb, A., y Kashif, R. (2012). La relación óptima del ciclo de conversión de efectivo con Tamaño de la empresa y la rentabilidad, 2(4), 189-203.

Muñoz, E. (2013). Propuesta de un modelo de Gestion y Control Financiero de corto plazo de la empresa Auto Centro Santa Ana AC S . A . Universidad de Costa Rica.

Nazir, M. I., Khan, M. A., y Khan, M. A. (2016). El impacto de la gestión del capital en empresas del rendimiento financiero: Evidencia de Pakistán, 6(3), 1097-1105.

Nguyen, T. L. P., y Sundaresan, M. (2018). Los efectos de la conversión en efectivo Ciclo sobre rentabilidad: Una penetración en la Agricultura y las industrias alimentarias en Tailandia, 11(1), 97-120.

Nobanee, H. (2018). Gestión de Operaciones y la rentabilidad de la firma: Un ciclo óptimo de conversión en efectivo.
Ponce, M. (2014). Indicators Impact' S of Inventory Control in the Supply Chain.

Porlles, J., Quispe, C., y Salas, G. (2013). Pronóstico financiero: métodos rápidos de estimación del fondo de maniobra o capital de trabajo estructural - Caso de una empresa comercial. Industrial Data, 16(1), https://doi.org/10.15381/idata.v16i1.298 6

Sánchez, P. (2011). Análisis financiero y su incidencia en la toma de decisiones de la empresa vihalmotos. universidad Tecnica de Ambato.

Valero, D. (2013). Evaluacion de la Liquidez y su afectacion a los estados financieros en la empresa ASF en el periodo 2010 2011. Universidad de Guayaquil.

Yera, R. (1999). Administración estratégica del efectivo. Universidad Autonoma de Nuevo Leon.

Zakari, M., y Saidu, S. (2016). El impacto de Efectivo ciclo de conversión en la rentabilidad de la empresa : Evidencia de Empresas de Telecomunicaciones de Nigeria Listed, 4(6), 342-350. 